mean the journal will fill a niche in a crowded field. For example Vol. 2, No. 1 consists of a superb bibliography of thousands of references compiled from a Westinghouse database. The index to keywords, authors, subjects and chemical element compositions is especially informative and useful. This source, in accessible, compact journal form, may well justify the cost of a trial subscription.

Featured articles in Journal of Superconductivity have included an outstanding, comprehensive review of heatcapacity measurements on cuprate compounds, and discussions of some technological applications. Contributions on magnetohydrodynamic ship propulsion, circuits, electron accelerators, logic devices and power applications are pitched at an introductory level, and should appeal to those involved in technology. The papers on sample growth and processing, with their exquisite colour photographs, exemplify the high quality of production. On average, the articles are brief and the first few issues do not pose a dire threat to library shelf space.

A committee of 39 distinguished scientists edits the International Journal of Modern Physics B, which covers condensed matter, statistical and applied physics. Another 28 experts oversee the section "Rapid Communications in High $T_{\mathrm{c}}$ Superconductivity". A broad geographical distribution of authors from Australia, Bahrain, Belgium, Brazil, Bulgaria and 19 other countries is evident in the handful of journals made available for review. In a similar vein, the supplement Modern Physics Letters $B$ includes rapid communications and a section of "Brief Reviews" which exhibits short (typically three-page), less-technical summaries of select specialities.

Materials microfaults are the mainstay topic for Superconductor Science \& Technology. Grain boundaries, oxygen defects and other imperfections need to be overcome for large-scale technology, and contributors examine the detailed morphology of samples in view of their influence on critical currents and other properties. Research on film-preparation techniques is neatly presented, with a critical examination of the prospects of the near-term applications in microwave detection and other signal-processing devices.

As the field matures, the emphasis on haste should yield to traditional values. It is a pleasure to find some interesting articles in all of the journals reviewed here. But their future success will be determined by the careful enforcement of standards, as well as by the setting of goals that complement rather than compete with those of established journals.

John Ruvalds is in the Department of Physics, University of Virginia, Charlottesville, Virginia 22901, USA.

\section{Communicating in a new era}

\section{Alan Windle}

Materials Science and Engineering B: Solid-State Materials for Advanced Technology. Editors M. Balkanski, K. Kamimura and S. Mahajan. Elsevier Sequoia. 12/yr. SwFr. 840.

Advanced Materials. Editor Peter Gölitz. VCH. 12/yr. DM 298, £115, \$190 (institutional); DM 98, £42, $\$ 79$ (personal).

MATERIALS Science and Engineering B: Solid-State Materials for Advanced Technology was launched in August last year. If it hadn't been for the advent of high- $T$. superconductivity, one wonders whether the publishers would have dared - a new

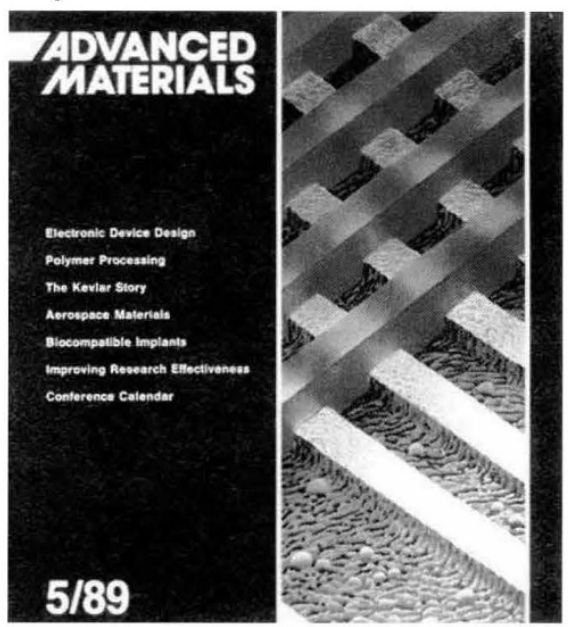

journal, in an otherwise fairly mature field which is already quite well provided for, is a risky venture.

Yet there is a clear professionalism about Solid-State Materials. It is a traditional, even classy, publication. One can play the name game, and try to guess its contents from the title, or perhaps from the editorial statement of scope inside the front cover. A better guide is the papers it has attracted to date, which deal almost exclusively with inorganic materials and concentrate most frequently on the relationship between structure and electronic properties. There is a good blend of well-established solid-state physics and solid-state chemistry, bound together by contributions which focus on the production of optical and electronic materials. The journal also provides a most appropriate environment for the 20 per cent or so of its papers which deal with the new superconductivity.

Authors seem to be treated well. The time between acceptance of papers and publication is averaging around six months, and there is every indication that this performance will be maintained. The quality of the reproduction of diagrams is high, and the micrographs come across adequately, although they are usually disciplined into single columns where their comparatively small size means that they lack impact, if not clarity. There are also 25 reprints for free.

For 1989, the journal bills itself as a quarterly, although one can but puzzle over the numbering system. The rather thin issue of December 1988 is designated $3-4$, whereas the first to appear this year (devoted to the proceedings of a conference on deep inplants) was numbered 1-3, which meant that number 4 appeared in April! Indeed, the slightly bizarre numbering is associated with a lack of uniformity both in publication dates and the thickness of the offering. Of course this may settle with time, and any confusion will tend to be reduced on binding.

Here we have a solid and respectable journal.

Advanced Materials is a different type of publication altogether. It is a monthly, aimed at the materials scientist, whose subject has grown and diversified so dramatically over the past ten years. Although it is possible to be an expert in one corner of the discipline, there is the perennial need to remain informed across the whole field, and a need too to foster a professional identity. If journals are to unify materials research rather than segregate it into subdisciplines, they must somehow represent the various components without becoming unwieldy.

Advanced Materials seems to have found this niche. It is a heady mixture of essays, review and research communications, news and conference data, and it works admirably. The emphasis is on new materials, and there is a fair balance across the component subjects of metallurgy, ceramics, composites and polymers. It is perhaps the journal one would most like to have delivered to a busy desk - the articles can be read in a few minutes, yet they communicate at the highest level and reflect the perception of their often-distinguished authors. One can of course quibble at the presentation; the paper used is too thin, for example, the print showing through from the other side of each page.

There is currently some basic rethinking, among materials scientists at least, as to how research should best be published, and the German community is among the leaders in this respect. We may be seeing the end of the traditional research paper, which could be superseded by a combination of rapid communication of results from laboratory to print with periodic state-of-the-art reviews. Advanced Materials is a part of this new era, and that is perhaps the underlying reason why it is exciting.

Alan Windle is in the Department of Materials Science and Metallurgy, University of Cambridge, Pembroke Street, Cambridge CB2 3QZ, UK. 